\title{
El acceso a la universidad en estudiantes con discapacidad: la influencia de la orientación educativa en la elección de estudios de grado
}

\author{
José Luis LOPEZ-BASTIAS, Ricardo MORENO-RODRIGUEZ \\ y Rosa ESPADA-CHAVARRIA
}

Universidad Rey Juan Carlos

(Recibido el 25 de Julio de 2018, Aceptado el 13 de Abril de 2020)

RESUMEN: El número de alumnos con discapacidad que decide matricularse en estudios universitarios de grado ha aumentado durante los últimos años. No obstante, si bien es verdad que la inclusión educativa ha favorecido la calidad y la permanencia de éstos en niveles educativos anteriores, la proporción de estudiantes con discapacidad que accede a la universidad sigue siendo inferior si es comparada con el resto del alumnado. El presente trabajo analiza la influencia que ha ejercido la orientación académica en la elección de es- tudios de grado en estudiantes con discapacidad que actualmente estudian en la Universidad Rey Juan Carlos. Para ello, se ha seguido una metodología descriptiva post-facto y se ha administrado un cues- tionario Ad Hoc sobre una muestra de 82 estudiantes con discapacidad, todos ellos registrados en la Unidad de Atención a Personas con Discapacidad y Necesidades Educativas Especiales de dicha uni- versidad. Los resultados muestran la aparición de otros factores, considerados como más importantes a la acción del orientador educativo en la elección de estudios universitarios. A su vez, se considera que la orientación académica llevada a cabo en los centros educativos de origen se realizó en momentos puntuales y se tuvo en cuenta, de manera prioritaria, otros factores diferentes a los del área de intereses y valores de los estudiantes. Se constata la necesidad de colaboración entre los centros universitarios y los centros de educación secundaria para favorecer la transición entre centros de Educación Secun- daria y la Universidad.

Palabras clave: discapacidad; acceso a la universidad; orientación educativa; educación inclusiva.

Access to University by Disabled Students: the Influence of Educational Guidance in the Choice of Degree Studies

\begin{abstract}
The number of students with disabilities choosing to enroll for university studies has increased over the last years. Nevertheless, even though it is true that educational inclusion has favoured the quality and permanence of this group of students in obligatory secondary and higher secondary education levels, that is, Compulsory Secondary Education and higher secondary education; it should
\end{abstract}


also be recalled that the ratio of people with disabilities who enter university is still tiny in comparison to the rest of students. This research sets out to analyze the influence of academic guidance on the choice of a university degree by students with a disability who are currently studying at Rey Juan Carlos University. A post-facto descriptive methodology was followed and an Ad Hoc questionnaire was administered on a sample of 82 students with disabilities, all of them registered in the Unit for Attention to People with Disabilities and Special Educational Needs of the same university. The results show the appearance of unknown fundamental factors which are relevant to the Guidance Action Plan, carried out by educational centers in students' countries of origin. This study took place in specific situations taking into account, other different factors apart from the interests and values of students. There is also a need for collaboration between universities and secondary schools to promote the transition between institutions and universities.

Keywords: Disability, Access to Higher Education, Transition Educational Guidance.

Correspondencia: José Luis López Bastías. Paseo de los Artilleros s/n. 28032. Madrid. E-mail: joseluis. lopez@urjc.es

\section{Introducción}

La Universidad es entendida como uno de los espacios formativos mas adecuados para favorecer la inclusión social y laboral de las personas con discapacidad (Espada, 2015; Peralta, 2007). La Ley Orgánica 4/2007, de 12 de abril, por la que se modifica la Ley Orgánica 6/2001, de 21 de diciembre, de Universidades, en cumplimiento con lo dictado por la Convención sobre los Derechos de las Personas con Discapacidad de 2006, pretendió impulsar la accesibilidad y la igualdad de oportunidades de las personas con discapacidad dentro del entorno universitario. El Real Decreto 412/2014, de 6 de junio, por el que se establece la normativa básica de los procedimientos de admisión a las enseñanzas universitarias oficiales de Grado, ya recogió y aportó diferentes medidas con el fin de favorecer el acceso a las enseñanzas de grado bajo el prisma de la igualdad de oportunidades, tanto a la hora de realizar las pruebas de acceso como en el momento de realizar la preinscripción y matriculación, mediante la recogida del sistema de reserva del 5\% de plazas para estudiantes con una discapacidad reconocida del $33 \%$ o superior. Por su parte, la Estrategia Europa 2020 también señala el papel que juegan sistemas de Educación Superior para impulsar el acceso de los estudiantes de los grupos que puedan ser más vulnerables, entre los que se encuentran los estudiantes con discapacidad.

Con todo esto, son diferentes los autores que consideran la última década como una etapa clave para la formación universitaria de las personas con discapacidad debido, entre otros factores, al aumento de este tipo de estudiantado (Álvarez-Pérez, Alegre de la Rosa y Lopez-Aguilar, 2015; Figuera y Coiduras, 2013; Biewer et al, 2015; Moriña, López-Gavira y Morgado, 2017). No obstante, tanto a nivel nacional como internacional, el número de estudiantes con discapacidad sigue teniendo baja representación en la Educación Superior (Lang, 2015). Se observan diferentes factores que pueden estar limitando el acceso del alumnado con discapacidad. En el contexto internacional, la falta de colaboración entre las instituciones de Educación Secundaria y la Universidad en la transición se torna como una de las principales barreras. Wadlington, De Ornellas y Scott (2017), en un estudio realizado en 408 universidades de los Estados Unidos, 
señalan que todavía existe una falta de consistencia en la colaboración con instituciones de Educación Secundaria y en el uso de planes de transición a la Universidad, por parte de los servicios de discapacidad. Otros autores también incidieron en esto último, entendiendo como uno de los factores clave para el acceso y permanencia de los estudiantes con Trastorno del Espectro del Autismo (TEA), la coordinación entre los distintos servicios educativos destinados a la transición entre etapas (Alverson, Lindstrom y Hirano, 2015; Anderson, Carter y Stephenson, 2017; Bakker, Krabbendam, Bhulai, y Begeer, 2019). Lambe et al., 2019).

Por su parte Al-A'dra (2016), en un estudio desarrollado en el contexto de Jordania, también encontró barreras en el acceso a la universidad por parte del estudiantado con discapacidad, debido a la deficitaria orientación académica y a las trabas administrativas. Similares fueron los resultados de Fajzrahmanova (2015) y Yarskaya-Smirnova y Zaitsev (2019), pero en el contexto ruso.

Por lo que respecta a España, en 2012, la cifra de personas con discapacidad que poseían un título universitario se situaba en el 1,14\%, mientras que entre las personas sin discapacidad era del 16,17\% (Instituto Nacional de Estadística, 2012). Actualmente, aunque no se ha vuelto a realizar un estudio de estas características, la Fundación Universia, a través del "IV Estudio sobre Universidad y Discapacidad", llevado a cabo durante el curso 2017/2018 en 75 universidades españolas, indicaba que el total de estudiantes con discapacidad se sitúa cerca del 1,5\% sobre el total de estudiantes (Fundación Universia, 2019).

En el contexto nacional, la elección de estudios universitarios en estudiantes con discapacidad puede estar condicionada por diferentes factores. Las actividades de atención al estudiantado con discapacidad en la universidad se centran más en la permanencia que en la promoción de estudios, siendo escasa la colaboración entre los centros de Educación Secundaria y los servicios de atención al estudiantado con discapacidad en el establecimiento de planes de transición para este tipo estudiantes (Galán-Mañas, 2015). Relacionado con esto último, y aunque puede que la mayor parte de los estudiantes elijan el grado universitario que van a estudiar movidos por un sentimiento de vocación, la discapacidad también puede ser un factor que influencia su situación. En este sentido, la falta de planes de orientación y de transición de una etapa a otra pueden resultar en un primer año duro para el estudiante (Moriña y Cortés, 2017). Así, se pueden dar repercusiones negativas de diferente índole. La ausencia de orientación puede desembocar en una elección de estudios insatisfactoria, la cual puede repercutir en el abandono, generando un coste de recursos perdidos en términos de tiempo, esfuerzo y dinero, tanto para el interesado como para la propia universidad. Pero no solo eso, una elección de estudios inadecuada también puede generar efectos negativos sobre la salud psicológica de la persona, como ansiedad o alteración de la autoestima (Sánchez 2001).

En este aspecto, es importante redirigir el sentido de la orientación y para ello es importante conocer bien hacia dónde y hacia qué se orienta, y hacer un análisis de la realidad que permita comprenderla e interpretarla (Martinez-Clares, Pérez-Cusó y Martínez Juárez, 2014).

Con toda esta información, sin embargo, son escasos los estudios actualizados que ahonden en esta temática, sobre todo en el sistema educativo español. Con el fin de continuar aportando evidencias empíricas en esta materia, este trabajo presenta los resultados obtenidos a partir del caso de la URJC. 


\section{Metodología}

\section{Muestra}

La muestra ha estado compuesta por 82 estudiantes universitarios que cursan estudios de grado en la URJC, los cuales se han identificado como personas con discapacidad y forman parte de la Unidad de Atención a Personas con Discapacidad de la misma universidad.

\section{Diseño y procedimiento}

Se llevó a cabo un cuestionario Ad Hoc, denominado como Cuestionario para el Análisis de la Influencia del Orientador Educativo en el Acceso a Estudios de Grado Universitario en Alumnado con Discapacidad (Anexo I). Los ítems del instrumento han sido realizados a partir de una amplia revisión bibliográfica sobre el tema y siguiendo la técnica Delphi. Se ha elegido este tipo de diseño por considerar que es una herramienta especialmente utilizada en la evaluación de programas, en la toma de decisiones, así como en situaciones de análisis prospectivos y retrospectivos, que pretende poner de acuerdo a personas expertas que participan y conocen bien un determinado sector (Echeita, Simón, Márquez, Fernández y Moreno, 2017; León y Montero, 2015).

El cuestionario está formado por 17 ítems, agrupados en dos dimensiones: recogida de datos sociodemográficos e influencia de la orientación educativa en el ingreso de los estudios de grado. Además, el cuestionario cuenta también con una pregunta final, de carácter abierto, sobre el proceso de orientación vivido en la anterior etapa educativa y la influencia en la elección de estudios universitarios (Tabla 1).

Tabla 1. Áreas y Factores del Cuestionario para el Análisis de la Influencia del Orientador Educativo en el Acceso a Estudios de Grado Universitarios en Alumnado con Discapacidad

\begin{tabular}{ll}
\hline Áreas & Factores \\
\hline Datos sociodemográficos & Edad \\
Sexo \\
Localidad de residencia \\
Estudios que cursa en la actualidad \\
Forma de acceso a los estudios actuales \\
Modalidad de la titulación \\
Curso \\
Tipo de discapacidad \\
Grado de discapacidad (\%) \\
Orientación recibida \\
Recursos disponibles en la orientación \\
Influencia ejercida por la orientación en la elec- \\
ción del tipo de estudios. \\
Influencia ejercida por la orientación en la elec- \\
ción final de grado universitario \\
Influencia ejercida por la orientación en la elec- \\
ción final de Universidad. \\
\hline
\end{tabular}


La información de este cuestionario fue recogida durante los meses de enero y febrero de 2018 (coincidiendo con el inicio del segundo cuatrimestre) y se administró una sola vez por participante. Se desarrolló un formato de la encuesta en papel y otro virtual, mediante la herramienta "Google Docs". La localización de los sujetos se llevó a cabo mediante la Unidad de Atención a Personas con Discapacidad con el fin de asegurar la confidencialidad y la protección de datos.

\section{Resultados}

Se han llevado a cabo dos tipos de análisis en el tratamiento de los datos:

a. De tipo descriptivo: medias, modas y datos porcentuales, mediante tablas de contingencia y gráficos en los que se cruzan las distintas variables de la investigación.

b. Cualitativo: en el que se analizan las respuestas libres de los estudiantes con discapacidad en contestación al ítem abierto incluido en el cuestionario. A través de un análisis observacional se encontraron 4 categorías, relacionadas con la presencia de orientación educativa, la coordinación entre diferentes etapas en materia de orientación educativa y la vivencia en la matriculación de estudios universitarios.

La muestra se distribuye en 82 participantes, de los cuales 44 son mujeres $(53,66 \%)$ y 38 son hombres (46,34\%). La mayoría (96,34\%), viven dentro de la Comunidad de Madrid, aunque se ha contado con la participación de un estudiante de Castilla la Mancha, otro de las Islas Canarias y otro de Euskadi. La edad media es de 29 años, siendo el rango de edad global de los encuestados entre los 18 y los 64 años.

Respecto a la rama de conocimiento de la carrera que están estudiando y el curso en el que se encuentran, los principales datos se recogen en las figuras 1 y 2.

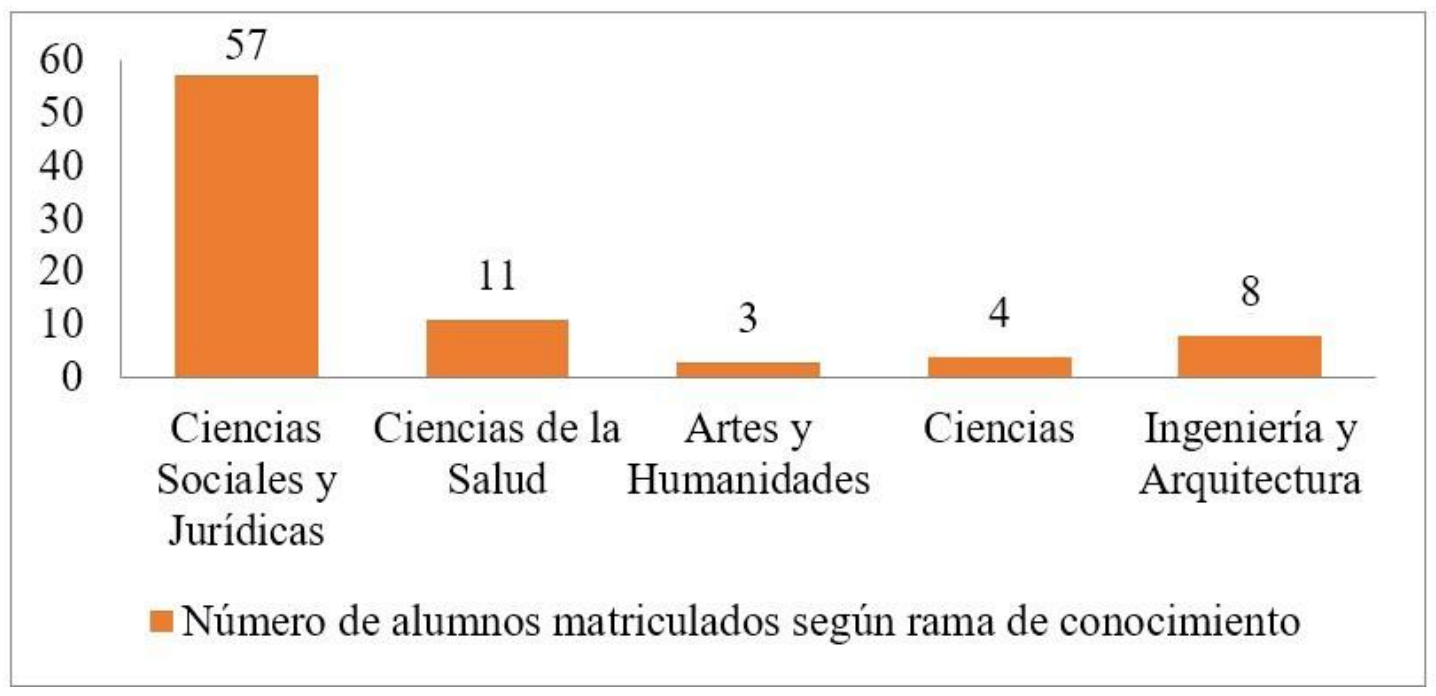

Figura 1. Número de alumnos matriculados según rama de conocimiento 


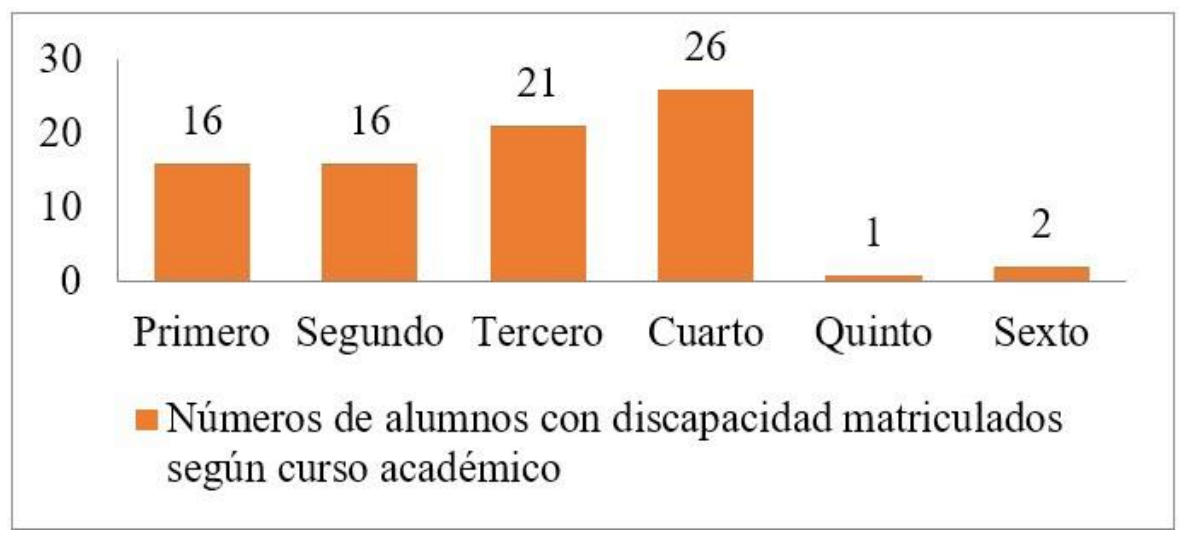

Figura 2. Número de alumnos con discapacidad matriculados en función del curso académico.

Otro dato que merece la pena destacar es el de la modalidad de la titulación en la que están estudiando. De los 82 participantes, 73 de ellos $(89,02 \%)$ estudian una modalidad presencial, mientras que el resto, 9 (10,98\%), han optado por una opción semipresencial u online.

En cuanto al tipo de discapacidad de los estudiantes, un 62,20\% presenta discapacidad física, un 17,07\% discapacidad psíquica, un 3,66\% discapacidad visual, un 9,02\% discapacidad visual, un 2,44\% discapacidad intelectual y un 4,88\% tiene más de una discapacidad (pluridiscapacidad).

Por otro lado, el grado de discapacidad más frecuente entre los estudiantes es del $33 \%$, situándose en un grado promedio de discapacidad del 56,22\%.

De los 82 alumnos, 42 de ellos $(51,21 \%)$ recurrieron en primer lugar a internet como forma de orientación para su futuro académico; 11 de ellos $(13,41 \%)$ recurrieron a sus familiares; 7 de ellos a la figura del orientador (8,53\%); 5 a la figura de sus amigos (6,09\%); 3 recurrieron en primer lugar a ferias educativas como "AULA", y el resto, 14, $(20,76)$ acudieron a otras fuentes (jornadas de puertas abiertas en universidades, folletos informativos y servicios de orientación de las diferentes universidades).

Por su parte, como se puede apreciar en la figura 3, la fuente más importante considerada por los alumnos en la elección de los estudios que están cursando actualmente han sido internet, seguido de los familiares y, en tercer lugar, la figura del orientador educativo.

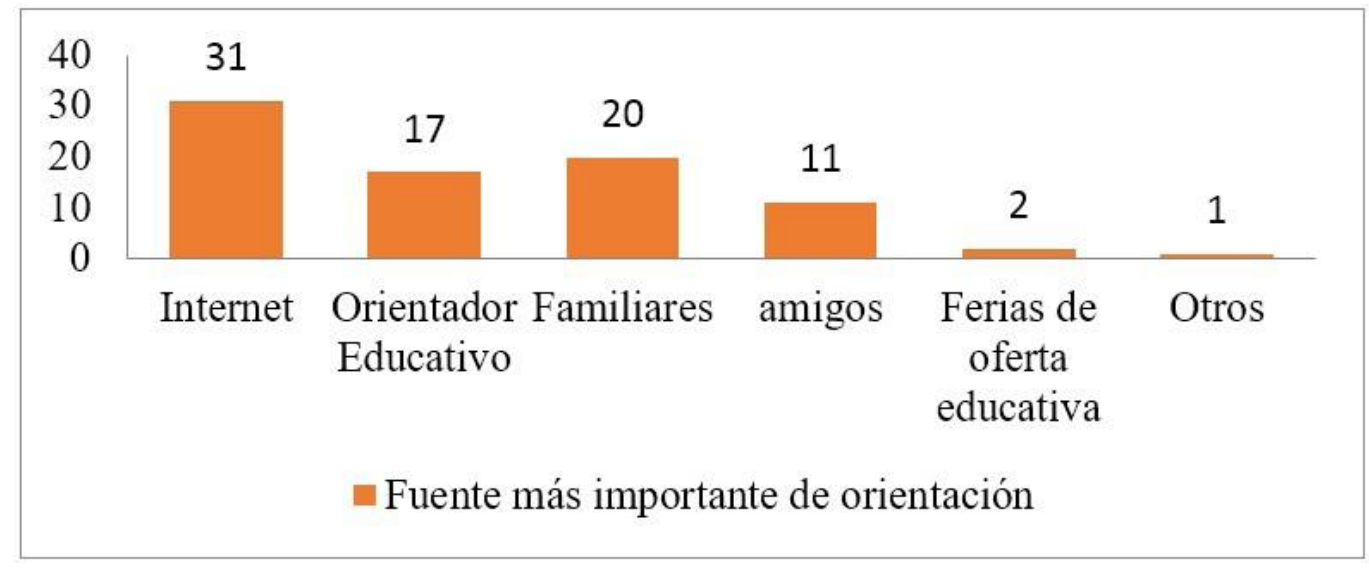

Figura 3. Fuente considerada como más importante en la orientación académica 
Con el fin de concretar más el objeto del estudio, la influencia del orientación académica y profesional en la elección de estudios universitarios en estudiantes con discapacidad de la URJC, a los 82 participantes se les realizó la pregunta de si habían recibido algún tipo de orientación académica en su anterior centro educativo. De los 82 sujetos, 28 de ellos $(34,14 \%)$ indicaron que sí habían recibido algún tipo de orientación académica para la elección de sus estudios, mientras que 54 aseguraban que no habían recibido ningún tipo de orientación $(65,85 \%)$

De los 28 alumnos que sí recibieron orientación académica relacionada con la elección de estudios superiores, una gran parte de ellos fueron orientados a estudios de grado (tabla 2).

Tabla 2. Opción Educativa Recomendada en la Orientación Recibida

\begin{tabular}{lcc}
\hline Opción educativa & Número & $\%$ \\
\hline Estudios Universitarios & 23 & 82.14 \\
Formación Profesional de grado superior & 2 & 7.14 \\
Formación Profesional de grado medio & 3 & 10.71 \\
Otros & 0 & 0 \\
\hline
\end{tabular}

Por otra parte, de los 28 alumnos que sí recibieron orientación académica, 10 de ellos $(35,71 \%)$ indican que sí obtuvieron asesoramiento por parte del orientador de su anterior centro educativo a la hora de escoger el grado que cursan actualmente, mientras que 18 indicaron no haber recibido ningún tipo de información en este sentido $(64,29 \%)$.

En lo referente a la influencia que el orientador había tenido a la hora de la elección personal de los estudios que están cursando actualmente, de los 28 sujetos que sí recibieron orientación, 18 de ellos $(64,28 \%)$ consideran que no hubo ninguna influencia por parte del orientador; 4 afirman que hubo algo de influencia (14,29\%), 3 indicaron que la influencia fue moderada $(10,71 \%)$ y otros tres respondieron que la influencia del orientador fue notable $(10,71 \%)$.

Del grupo que sí recibió orientación académica, tal y como se muestra en la tabla 3, se indicaron que los factores principales que más había valorado el orientador a la hora de elegir entre la oferta académica habían sido, en primer lugar, las futuras salidas laborales y el salario $(35,71 \%)$; en segundo lugar la cercanía del domicilio al centro de estudios $(21,43 \%)$; en tercer lugar el sistema de valores e intereses del estudiante $(17,85 \%)$ y en cuarto lugar el conjunto de capacidades $(14,28 \%)$.

Tabla 3. Factores Tenidos en Cuenta en la Orientación hacia Estudios Universitarios de Grado

\begin{tabular}{lcc}
\hline Factores tenidos en cuenta en la orientación & Número & \% \\
\hline Futuras salidas laborales y sueldo & 10 & $35,71 \%$ \\
Cercanía del centro de estudios respecto del domicilio & 6 & $21,43 \%$ \\
Intereses y valores del estudiante & 5 & $17,86 \%$ \\
Capacidades del estudiante & 4 & $14,28 \%$ \\
Otras (sin especificar) & 3 & $10,71 \%$ \\
\hline
\end{tabular}


En lo referente a la influencia final de la orientación académica recibida, en la elección final de los estudios que cursan actualmente, 15 de los alumnos que sí habían recibido orientación, respondieron que no hubo nada de influencia $(53,57 \%), 4$ aseguraron que fue escasa $(14,29 \%), 6$ respondieron que fue moderada (el 21,43\%) y 3 de ellos afirmaron que la influencia fue notable $(10,71 \%)$.

Por último, de los 28 sujetos que sí recibieron orientación académica en la elección de estudios, 17 consideraron que ésta no había tenido influencia en la elección final de la Universidad en la que están estudiando (60,71\%), 5 indicaron que había influido muy poco $(17,86 \%), 4$ de ellos $(14,29 \%)$ señalaron que la influencia en la elección de universidad fue moderada y dos de ellos consideraron que la influencia fue notable $(7,14 \%)$ (figura 4$)$.

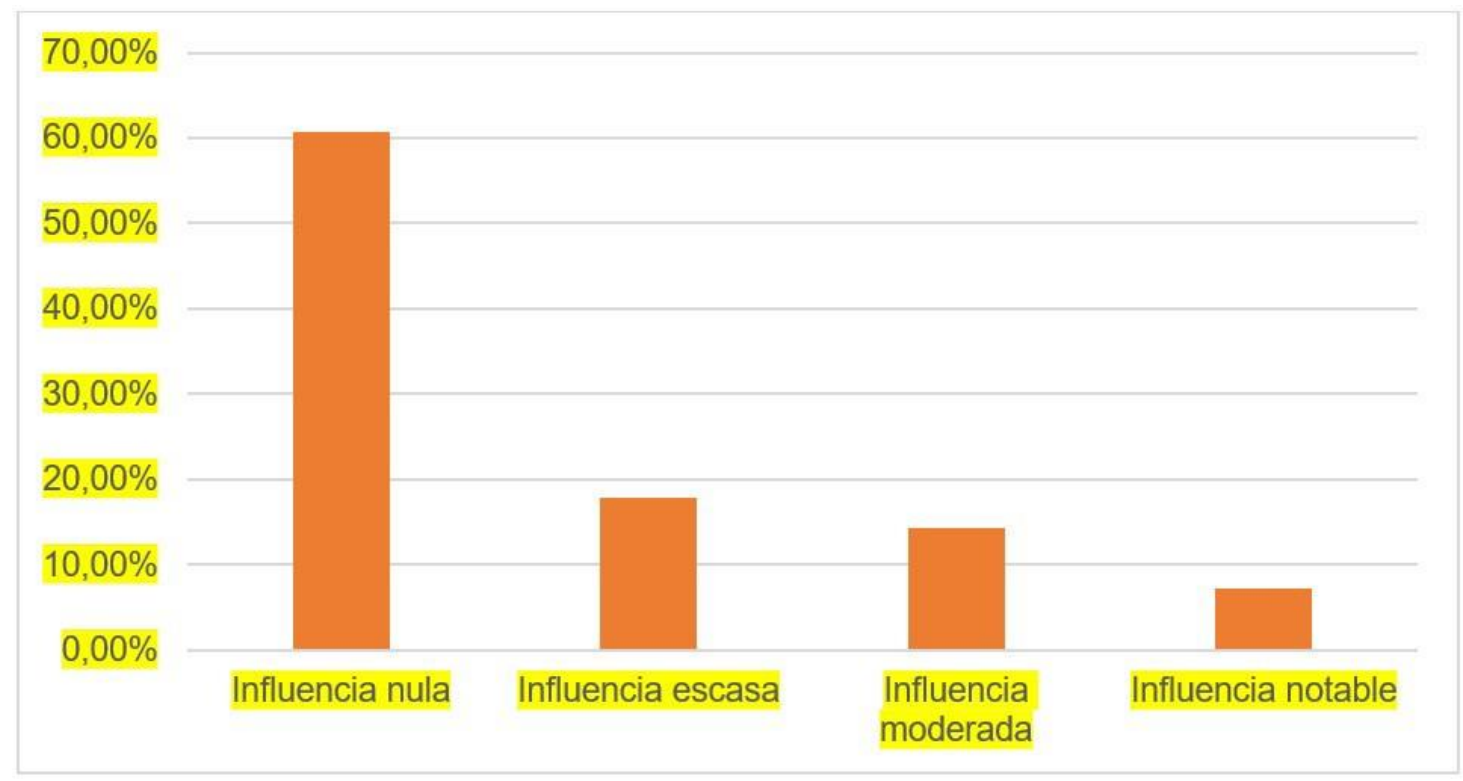

Figura 4. Influencia de la orientación educativa en la elección final de universidad

Del análisis cualitativo correspondiente al ítem de respuesta abierta se desprenden los siguientes datos, priorizados:

- La orientación académica recibida es insuficiente. Parte de los estudiantes refieren no haber tenido orientación académica en la elección de estudios universitarios. En los casos en los que se da se produce de manera puntual durante $2^{\circ}$ de Bachillerato. Dicha orientación consiste en la visita a ferias educativas como AULA y a universidades cercanas al centro educativo.

- Falta de coordinación y colaboración entre centro de Educación Secundaria y la Universidad. Los estudiantes con discapacidad indican el desconocimiento de servicios de orientación en las universidades y servicios propios para la atención a las necesidades educativas especiales. También indican que, en caso de conocerlos, suelen ser en las universidades en las que han realizado las pruebas de acceso. A menudo desconocen la existencia de este tipo de servicios incluso durante el primer año de estudiosuniversitarios.

- Mejorar la orientación para conocer mejor las opciones educativas con las que cuentan con base a los intereses y a las capacidades de los estudiantes. De las respuestas abiertas 
se recoge inseguridad del estudiante en el momento de matricularse en el grado universitario como parte del desconocimiento de las demandas que supondrán cada una de las materias y a la incertidumbre respecto al acceso a posibles adaptaciones.

\section{Discusión y conclusiones}

Se observa una tendencia en el tipo de estudiantado que decide cursar estudios universitarios de grado, siendo la discapacidad física y $(62,9 \%)$ y la rama de Ciencias Sociales y Jurídicas $(69,5 \%)$ las más representadas. Esto se mantiene en sintonía con un estudio de características similares realizado por Álvarez-Pérez et al. (2015). El perfil mayoritario puede ser el de los estudiantes con discapacidad física dado que este tipo de estudiantes presentan hándicaps de carácter más leves y, por lo tanto, no incapacitantes. En lo concerniente al área de conocimiento de los grados, la elección de estudios en la rama de Ciencias Sociales y Jurídicas, puede deberse a que los recursos específicos que necesitan para participar en el ámbito educativo son menores. Sin embargo, a la luz de estos resultados, puede estar dándose el caso de estudiantes con diferentes tipos de discapacidad que no lleguen a finalizar los estudios secundarios; que no estén siendo orientados hacia estudios universitarios o estén siendo redirigidos hacia otros grados diferentes de los que mejor se adaptan a su sistema de intereses y capacidades.

Por tanto, es necesario seguir trabajando para favorecer el acceso, la permanencia y la consecución de logros por parte del colectivo de personas con discapacidad en el entorno universitario. Para ello, en primer lugar, se hace necesario favorecer la finalización, con garantías de éxito, de los estudios en etapas educativas anteriores. De esto se desprende que sea necesario un profesorado formado, tanto en materia de discapacidad, como en didácticas que favorezcan la inclusión educativa. De todas ellas, y en línea con el objetivo nº 4 de la Agenda 2030, el Diseño Universal para el Aprendizaje (DUA) es una de las herramientas más eficaces en la generación de prácticas docentes que fomenten una educación personalizada y de calidad (Elizondo, 2020).

Por otro lado, la orientación debe dejar a un lado el papel de informar o condicionar la toma decisiones para pasar a ofrecer la ayuda necesaria que anime al estudiante a descubrirse como un ciudadano responsable, motivado y productivo (Vélaz de Medrano, 2011; Martínez-Martínez, Zurita y Castro-Sánchez, 2016). Además, una buena orientación debe acompañar a la persona a alcanzar su autorrealización a través del autoconocimiento de las fortalezas, oportunidades, amenazas y debilidades (Martinez-Clares, et al., 2014).

Los resultados también indican la importancia que adquiere internet y las nuevas tecnologías en esta materia. Así, una de las funciones clave del orientador puede ser la de propiciar un adecuado uso de las redes por parte de los destinatarios de la orientación (Oliva 2015; Rey, Pérez y Santalla, 2013).

De igual forma, también hay que poner de manifiesto el papel que debe jugar la Universidad en este proceso como agente continuador en la de formación de los estudiantes con discapacidad. Existen posibles carencias que desde el ámbito universitario nacional es necesario subvertir. Uno de los principales órganos promotores del acceso de estudiantes con discapacidad han de ser los servicios de apoyo al estudiante con discapacidad. El IV informe de la fundación Universia, sobre Universidad y Discapacidad, estima que cerca del 80\% de las universidades españolas ofrecen servicio de orientación preuniversitaria. Sin embargo, cerca de un $40 \%$ desco- 
nocen la existencia de estos servicios cuando ingresan en la universidad (Fundación Universia, 2019). Una posible solución a esto pasa por dar mayor visibilidad a este recurso, identificando como potencial beneficiario del mismo, no solamente al estudiante con discapacidad, sino también al conjunto de los Centros de Educación Secundaria. Deben establecerse lazos de colaboración entre los orientadores educativos y los técnicos responsables de los servicios de atención al alumnado con discapacidad. En línea con lo evidenciado por otros estudios en esta materia, dentro del contexto español, Figuera y Coiduras (2013) indican la debilidad en la transición entre los sistemas educativos que debería superarse fomentando la colaboración interinstitucional. De forma similiar, Álvarez-Pérez et al. (2015) también subrayaron la falta de programas de orientación e información para el acceso a estudios universitarios y recalcaron la necesidad de implementar medidas de apoyo para facilitar el proceso de aprendizaje, así como mejorar las políticas institucionales en materia de discapacidad.

Por otro lado, y con relación a los servicios de discapacidad, existe otra barrera que puede limitar la efectividad de la acción orientadora. Tanto su estructura como el perfil profesional de los técnicos que trabajan en ella es muy heterogéneo (Fundación Universia, 2019). De esta manera, dichos servicios no tienen por qué contar con profesional especializado en materia de orientación académica. Es importante que los servicios de atención al estudiante con discapacidad adquieran un carácter multidisciplinar en el que se ahonde en la función orientadora y que, además, permita un modelo de investigación-acción como forma de innovación y de mejora continua en esta materia. Todo esto, además, puede favorecer el carácter transversal de los servicios de discapacidad dentro de la comunidad educativa, superando su mero carácter informador y destinado a momentos puntuales.

\section{Limitaciones}

El estudio llevado adolece de una muestra los suficientemente amplia como para generalizar los resultados. Además, habría sido necesario homogeneizar la edad del grupo analizado como forma de asegurar que todo el alumnado accede a la universidad tras haber estudiado bajo la misma ley educativa.

\section{Referencias bibliográficas}

Al-A'dra, I.A. (2016). Challenges facing students with disabilities at the University of Jordan: a field study. Dirasat: Human and Social Sciences, 43, 2013-2032.

Álvarez-Pérez, P., De la Rosa, O., Aguilar. D. (2015). Transition and Access to higher education in students with specific educational needs: a diagnosis of the difficulties in adaptation. Research on University Teaching and Faculty Development, 3(3), 115-129.

Alverson, C., Lindstrom, L., y Hirano, K. (2015). High school to college: transition experiences of young adults with autism. Focus on autism and other developmental disabilities, 1-13. DOI: https://doi.org/10.1177/1088357615611880

Anderson, A. H., Carter, M., y Stephenson, J. (2017). Perspectives of university students with autism spectrum disorder. Journal of Autism and Developmental Disorders, 31(3), 27-15. DOI: https://doi.org/10.1007/s10803-017-3257-3

(C) Psy, Soc, \& Educ, 2020, Vol. 12(2) 
Bakker, T., Krabbendam, L., Bhulai, S., y Begeer, S. (2019). Background and enrollment characteristics of students with autism in higher education. Research in Autism Spectrum Disorders, 68. DOI: https://doi.org/10.1016/j.rasd.2019.101424

Biewer, G., Buchner, T., Shevlin, M., Smyth, F., Siska, J., Kanova, S., Ferrerira, M., TobosoMartin, M., y Rodríguez, S. (2015). Pathways to inclusion in European higher education systems. ALTER, European Journal of Disability Research, (9) 278-289. DOI: https://doi. org/10.1016/j.alter.2015.02.001.

Echeita, G., Simón, C., Márquez, C., Fernández, M., y Moreno, A. (2017). Análisis y valoración del área de educación del III plan de acción para personas con discapacidad en la Comunidad de Madrid (2012-2015). Siglo Cero, 48(1), 51-71.

Elizondo, C. (2020). Hacia la inclusión educativa en la universidad: diseño universal para el aprendizaje y la educación de calidad. Barcelona, España: Octaedro.

Espada, R. (2016). Madurez vocacional en estudiantes universitarios con discapacidad: orientación y formación en competencias transversales para la mejora de la empleabilidad y el desarrollo profesional. Tesis doctoral. Universidad de Salamanca, Salamanca, España. Recuperado de https:/gredos.usal.es/bitstream/handle/10366/128436/ DPE TP_EspadaChavarr\%C3\% ADa R_Ma dure zvoc acionalest udi ant es. pdf?sequence $=1 \&$ isAllowed $=y$

Fajzrahmanova, A. T., (2015). Organizational and Pedagogical Education Terms for Disabled Persons at Universities. The Social Sciences, 10, 444-448.

Ferreira, C., Vieira, MJ., y Vidal, J (2014). La atención a los estudiantes con discapacidad en las instituciones de educación superior. El caso de Cataluña. Revista de Investigación Educativa, 32(1), 139-157. DOI: https://doi.org/10.6018/rie.32.1.171711

Figuera, P., y Coiduras, J.L. (2013). The transition to university: An analysis in terms of the diversity of student voice. Revista de Educación, 362, 713-736. DOI: 10.4438/1988-592XRE-2013-362-247

Fundación Universia(2019). Universidad y Discapacidad: IVEstudio sobre el grado de inclusión del sistema universitario español respecto dela realidad de la discapacidad. Recuperado de:https:// www.fundacionuniversia.net/wp-content/uploads/2019/05/IVEstudio_Universidady Discapacidad_ACC.pdf

Galán-Mañas, A. (2015): Orientación a los estudiantes con discapacidad en la Universidad Española. Revista Española de Orientación y psicopedagogía, 26(1) 83-89.

Instituto Nacional de Estadística - INE. (2012) Encuesta de Integración Social y Salud 2012.

Lambe, S., Russell, A., Butler, C., Fletcher, S., Ashwin, C., y Brosnan, M. (2019). Autism and the transition to university from the student perspective. Autism, 23(6), 1531-1541. DOI: https://doi.org/10.1177\%2F1362361318803935

Lang, L. (2015). Responsibility and participation in transition to university-voices of Young people with disabilities. Scandinavian Journal of Disability Research, 17(2), 130- 143. DOI: https://doi.org/10.1080/15017419.2013.817355

León, O. y Montero, I. (2015). Métodos de investigación en Psicología y Educación (4ª ed). Madrid: McGraw-Hill. 
Martínez Clarés, P., Pérez-Cusó, F.J., y Martínez Juárez, M. (2014). Orientación Profesional en Educación Secundaria. Revista Electrónica Interuniversitaria de Formación del Profesorado, 17(1), 57-71. https://doi.org/10.6018/reifop.17.1.198841

Martínez-Martínez, A., y Zurita F. (2014). El rol que tiene la influencia familiar y su nivel académico en los itinerarios curriculares del estudiante de último curso. Educatio Siglo XXI, 32(2), 267- 286. DOI: https://doi.org/10.6018/j/202251

Moriña, A., Molina, V.M., y Cortés-Vega, M.D. (2017). Voices from Spanish With disabilities: willpower and effort to survive university. European Journal of Special Needs Education, 33(4), 481-494.DOI: https://doi.org/10.1080/08856257.2017.1361138

Peralta, A. (2007). Libro blanco sobre universidad y discapacidad. Madrid. España. Real Patronato sobre Discapacidad. Recuperado de: https://sapdu.unizar.es/sites/default/files/080326\%20 0856\%20Libro\%20Blanco.pdf

Oliva, C.R (2015) La utilización de las TIC en la orientación educativa: Un estudio exploratorio sobre la situación actual de uso y formación entre los profesionales de la orientación. Revista Española de Orientacion y Psicopedagogia, 26(3), 78-95. DOI: https://doi.org/10.5944/ reop.vol.26.num.3.2015.16402

Real Decreto 412/2014, de 6 de junio, por el que se establece la normativa básica de los procedimientos de admisión a las enseñanzas universitarias oficiales de Grado.

Repetto, E., Guillamón, J.R., Vélaz de Medrano, C., y Malik, B. (2002): Nuevas Tecnologías aplicadas a la orientación. En Bisquerra, R. (coord). Barcelona, Praxis.

Rey, E., Pérez, M., y Santalla, A. (2013). El uso de las Tecnologías de la Información y la Comunicación en la Orientación Educativa: explorando la familiaridad y preparación de los profesionales del ámbito en España. Revista Mexicana de Orientación Educativa, $10(24), 45-57$.

Sánchez, M. (2001). La orientación universitaria y las circunstancias de elección de los estudios. Revista de Investigación Educativa 19 (1) 39-61.

Sursock, A., y Smidt, H. (2010). Trends 2010: A decade of change in European Higher Education. Bruselas: EUA Publications. Recuperado de: https://eua.eu/downloads/publications/ trends $\% 202010 \% 20 \mathrm{a} \% 20$ decade $\% 20$ of $\% 20$ change $\% 20$ in $\% 20$ european $\% 20$ higher $\% 20$ education.pdf

Vélaz de Medrano, C. (2011). La orientación académica y profesional en la Educación Secundaria. En E. Martín, y I. Solé (Coords.), Orientación Educativa: Modelos y Estrategias de intervención (pp. 129-149). Barcelona, España: Ministerios de Educación-Graó.

Wadlington, C., De Ornellas, K., y Scott, S.R. (2017). Developing effective transition reports for secondary students: a nationwide survey of college and University disability Support Personnel. Exceptionality, 25(3), 207-215. DOI: https://doi.org/10.1080/09362835.2017.1 283623

Yarskaya-Smimova, V., y Zaitsev, D. (2019). The habitus of disability in the field of higher education. Zhurnal Issledovanii Sotsial'noi Politiki, 14(4), 585-600. 


\section{ANEXO}

\section{CUESTIONARIO PARA EL ANÁLISIS DE LA INFLUENCIA DEL ORIENTADOR EDUCATIVO EN EL ACCESO A ESTUDIOS DE GRADO UNIVERSITARIOS EN ALUMNADO CON DISCAPACIDAD}

Este cuestionario tiene como objetivo obtener los datos necesarios para valorar el nivel de orientación académica y profesional, recibida por los estudiantes con discapacidad de la Universidad Rey Juan Carlos, en la elección de sus estudios de grado. Los datos obtenidos en dicha en cuesta, totalmente anónima, únicamente serán utilizados con fines académicos.

Instrucciones: rellene sobre la línea la información solicitada.

Edad__ Titulación

Tipo de discapacidad*

Sexo_ Curso___ Grado de discapacidad (\%)

Lugar de residencia

*Física y/u orgánica, psíquica, sensorial/visual, sensorial/auditiva, intelectual

1. ¿Cuál es la vía de acceso por la que ha accedido al grado que está estudiando?

\begin{tabular}{ll}
\multicolumn{1}{c}{ Vía de Acceso } & Marque con un aspa \\
\hline Bachillerato y Formación Profesional & \\
Titulados universitarios & \\
Estudiantes mayores de 25 años & \\
Estudiantes mayores de 40 años \\
Estudiantes mayores de 45 años
\end{tabular}

2. ¿Ha recibido algún tipo de asesoramiento por parte del orientador de su anterior centro educativo a la hora de conocer la oferta educativa disponible?

$$
\text { SÍ___ NO_ }
$$

3. Considera que el asesoramiento por parte del orientador de su anterior centro educativo a la hora de conocer la oferta disponible ha sido (marque solo una opción)

\section{Marque con un aspa}

Inexistente

Escasa

Suficiente

Adecuada 
4. ¿A qué fuente ha recurrido en primer lugar para recibir información sobre la oferta educativa disponible? (Marque sólo una)

\section{Marque con un aspa}

Orientador educativo

Internet

Familiares

Amigos

Ferias de oferta educativa

Otros

5. ¿Cuál ha sido la principal fuente a la que ha recurrido para informarse sobre la oferta educativa disponible? (ordénelas según la importancia, siendo 1 la más importante y 6 la menos importante. No se pueden repetir opciones).

Marque con un aspa

Orientador educativo

Internet

Familiares

Amigos

Ferias de oferta educativa

Otros

6. En el caso de haber recibido asesoramiento por parte del orientador de su anterior centro educativo ¿Cuál fue la principal opción educativa recomendada? (marque sólo una opción).

\section{Marque con un aspa}

Estudios Universitarios

Formación Profesional de Grado Superior

Formación Profesional de Grado Medio

Otros

7. ¿Ha recibido algún tipo de asesoramiento por parte del orientador de su anterior centro educativo a la hora de elegir los estudios que cursa actualmente?
SÍ
$\mathrm{NO}$ 
8. En el caso de haber recibido asesoramiento por parte del orientador de su anterior centro educativo ¿Ha tenido alguna influencia en la elección personal de sus estudios?

\begin{tabular}{ll}
\cline { 2 - 2 } & Marque con un aspa \\
\hline Ninguna & \\
Insuficiente & \\
Moderada \\
Notable
\end{tabular}

9. En el caso de haber recibido asesoramiento ¿Considera que el asesoramiento recibido por parte del orientador de su anterior centro educativo en lo referente al grado que está estudiando actualmente ha sido:

\section{Marque con un aspa}

Inexistente

Escaso

Suficiente

Adecuada

10. Principalmente, a la hora de elegir el grado que está estudiando, el factor que más ha valorado, personalmente, ha sido (ordénelas por orden de preferencia siendo 1 la más importante y 5 la menos importante. No se pueden repetir opciones):

Las futuras salidas laborales y el salario

Se ajusta a mi área de intereses y valores

Se ajusta a mis capacidades

La cercanía del centro respecto al domicilio

Otras

11. Principalmente, a la hora de elegir el grado que está estudiando, el factor que más ha valorado el orientador de su anterior centro educativo ha sido (Ordénelas por orden de preferencia siendo 1 la más importante y 5 la menos importante.).

Las futuras salidas laborales y el salario

$\mathrm{El}$ área de intereses y valores

Se ajusta a mis capacidades

La cercanía del centro respecto al domicilio

Otras 
12. En el caso de haber recibido asesoramiento por parte del orientador de su anterior centro educativo ¿Consideras que se ajustaba a su área de intereses?

\section{Marque con un aspa}

Muy en desacuerdo

En desacuerdo

De acuerdo

Muy de acuerdo

13. En el caso de haber recibido asesoramiento por parte del orientador de su anterior centro educativo ¿Considera que se ajusta a sus capacidades?

\section{Marque con un aspa}

Muy en desacuerdo

En desacuerdo

De acuerdo

Muy de acuerdo

14. ¿Ha influido la opinión del orientador de su anterior centro educativo en la elección final del grado que está estudiando?

\begin{tabular}{ll}
\cline { 2 - 2 } & Marque con un aspa \\
\hline Nada & \\
Poco & \\
Moderado & \\
Notable & \\
\hline
\end{tabular}

15. ¿Ha influido la opinión del orientador de su anterior centro educativo en la elección final de la universidad en la que está estudiando?

Marque con un aspa

Nada

Poco

Moderada

Notable 
16. ¿Considera que la labor del orientador en materia de asesoramiento académica y profesional ha sido la adecuada?

\section{Marque con un aspa}

Muy en desacuerdo

En desacuerdo

De acuerdo

Muy de acuerdo

17. En esta pregunta puedes ampliar información que consideres importante relacionada con el proceso de orientación experimentado en la etapa previa al acceso a la universidad y tu valoración sobre la influencia de dicha orientación en la elección de los estudios de grado que cursas actualmente. 